\title{
Thermal Transitions of Liquid Crystalline Azoxydibenzoate Copolyesters
}

\author{
Naoyuki KoIDE, Ryo OHTA, and Kazuyoshi IIMURA \\ Department of Chemistry, Science University of Tokyo, \\ Kagurazaka, Shinjuku-ku, Tokyo 162, Japan
}

(Received February 27, 1984)

\begin{abstract}
The azoxydibenzoate copolyesters were obtained by the combination of azoxybenzene group as a mesogenic core with two different flexible chain lengths of oxyethylene unit. Their liquid crystalline behavior was studied by measuring of polarized microscopy, X-ray diffraction and differential scanning calorimetry. Smectic phase was observed for all the copolyesters. These copolyesters have a wider mesomorphic temperature range than that of corresponding homopolyesters.
\end{abstract}

KEY WORDS Azoxydibenzoate / Copolyester / Thermotropic Liquid Crystalline Polymer / Smectic Phase / Oxyethylene Unit /

Recently the discovery of ultra-high strength fibers from the nematic solution of aromatic polyamides has attracted a considerable interest in liquid crystalline polymers. ${ }^{1}$ With respect to the thermotropic liquid crystalline polymers, several types of polymers have been reported. One type has a comb-like polymer having a mesogenic or rigid molecular group in the side chain of polymer backbone. In the other type of polymers, a mesogenic group or rigid molecular group is incorporated into the polymer chain backbone. There are a wide variety of methods for obtaining thermotropic liquid crystalline polymers by the combination of different kinds of mesomorphic or rigid molecules with a flexible chain molecules. ${ }^{2}$

These polymers usually have very high transition temperatures from the solid to the liquid crystalline state. Many attempts have been carried out to obtain a polymer with a lower transition temperature. For the comb-like polymer, this goal has been achieved by introducing a flexible spacer between the main chain backbone and the mesogenic pendant group or by using a polysiloxane chain in the main chain backbone. ${ }^{3,4}$ It would also be possible to lower the transition temperature of the main chain type polymers by introducing a longer flexible molecular group between the rigid mesogenic molecules, but these main chain type polymers are not susceptible to show the liquid crystalline state. ${ }^{5,6}$ In the case of low molecular weight liquid crystalline materials, the mesomorphic temperature range is usually controlled by the mixing of the different ratio of the liquid crystalline components. The mesomorphic temperature range can be broadened, and lowered as a result of the formation of an eutectic mixture by mixing with some other low molecular weight liquid crystalline materials.

It would also be anticipated that the transition temperature from the solid to the mesophase of liquid crystalline polymer will be lowered by the copolymerization of the mesogenic unit with the combination of the different length of flexible parts.

This paper describes the synthesis and the phase diagram of the main chain type of azoxydibenzoate homo- and copolyesters containing the various combinations of di(oxyethylene) and tetra(oxyethylene) units having the following structures, $[\mathbf{I}]$ and $[\mathbf{I I}]$ : 
[I]

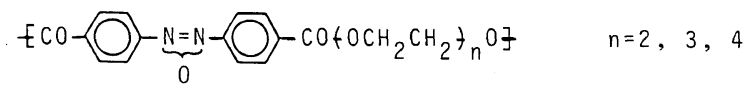

[II]

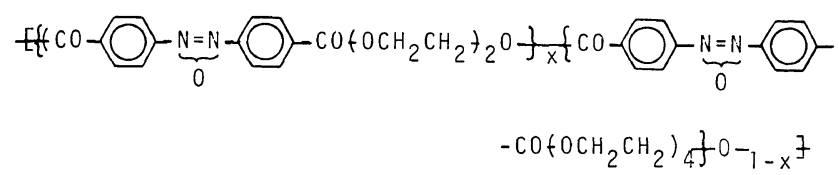

Poly[oligo(oxyethylene) azoxydibenzoate] [I] is abbreviated as POEAOB- $n(n=2,3$, and 4$)$, $n$ means the number of oxyethylene unit. Copolyester is also abbreviated as CoPOEAOB2-4- $x$ [II]. The number of 2 and 4 means the number of oxyethylene unit, and the molar \% of di(oxyethylene) unit in the flexible part of copolyester is shown by the letter $x$.

\section{EXPERIMENTAL}

$p, p^{\prime}$-Azoxydibenzoic acid was synthesized by reacting $p$-nitrobenzoic acid with sodium hydroxide in the presence of methanol according to Iida's azoxybenzene synthetic method. ${ }^{7}$ $p, p^{\prime}$-Azoxydibenzoyl chloride was obtained by refluxing the corresponding dicarboxylic acid with an excess amount of thionyl chloride in the presence of small amount of dimethylformamide. After $20 \mathrm{~h}$ reaction, unreacted excess amount of thionyl chloride was removed by vacuum distillation. The crude product was purified by recrystallization from the toluene solution. The melting point of the product was found to be $146^{\circ} \mathrm{C}$. From the IR and NMR spectra in DMSO- $d_{6}$, the structure of the purified product was confirmed.

Oligo(ethylene glycol)s from Tokyo Kasei were purified by a vacuum distillation with a small amount of sodium.

The copolymerization of azoxydibenzoate copolyester was carried out by a polycondensation reaction of $p, p^{\prime}$-azoxybenzoyl chloride with the mixture of the different ratios of diethylene glycol and tetraethylene glycol in $o^{-}$ dichlorobenzene at $179^{\circ} \mathrm{C}$. After removing the solvent at atmospheric pressure, furter poly- condensation was proceeded with heating under reduced pressure in order to get the higher molecular weight copolyester. The copolyesters dissolved in $p$-chlorophenol were purified by reprecipitating into methanol.

Inherent viscosities of the copolyesters were measured with an Ubbelode viscometer in $p$-chlorophenol at $43^{\circ} \mathrm{C}$. The molecular weight of the soluble polymer in tetrahydrofuran was estimated from the Gel Permeation Chromatography using a Toyo Soda model HLS- 802 at $40^{\circ} \mathrm{C}$. The standard polystyrenes were used for calibration.

DSC measurements were performed upon 5-10 mg samples with a Perkin-Elmer DSC1B calorimeter at a scanning rate of $10^{\circ} \mathrm{C}$ $\min ^{-1}$.

The optical texture of the samples was observed under the polarized microscope equipped with an Union optical MHS-3 type of heating plate.

X-Ray diffraction patterns were obtained with B-1 type of a Rigaku Denki diffractometer with $\mathrm{Cu} K_{\alpha}$ radiation using a nickel filter and GM counter.

\section{RESULTS AND DISCUSSION}

With increasing the flexible chain length, the mesomorphic temperature range became narrow and the transition temperature from the mesophase to the isotropic fluid $T_{\mathrm{m} \rightarrow \mathrm{i}}$ was lowered..$^{5}$ It was, however, important to check the molecular weight dependence of the transition temperature from the solid to the mesophase $T_{\mathrm{s} \rightarrow \mathrm{m}}$ and the mesomorphic temperature range $\left(\Delta T=T_{\mathrm{m} \rightarrow \mathrm{i}}-T_{\mathrm{s} \rightarrow \mathrm{m}}\right)$. Figure 1 shows 


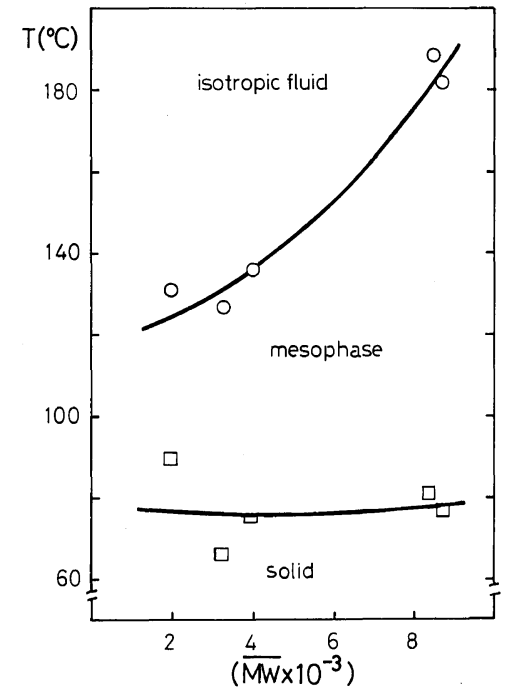

Figure 1. The transition temperature dependence on molecular weights in the range from 2000 to 8000 for POEAOB-3 polyester; $(\square)$, transition temperature on melting; ( $\bigcirc$ ), transition temperature on formation of the isotropic fluid.

the transition temperature of POEAOB-3 as a function of number average molecular weight in the range from 2000 to 8000 . The $T_{\mathrm{s} \rightarrow \mathrm{m}}$ was unchanged, while the $T_{\mathrm{m} \rightarrow \mathrm{i}}$ increased with increasing molecular weight. However, it was anticipated that the curve of $T_{\mathrm{m} \rightarrow \mathrm{i}}$ reached to a constant transition temperature at the high molecular weight above 8000 . This behavior has been confirmed by Blumstein et al. ${ }^{8}$ They have found that the $T_{\mathrm{m} \rightarrow \mathrm{i}}$ of polyester prepared from 4,4'-dihydroxy-2,2'-dimethylazoxybenzene and decandioic acid increased with increasing molecular weight, and reached to a constant value above approximately 10000 .

The transition temperature was found to be significantly influenced by the flexible chain length in the polyesters. For POEAOB- $n$ series, the $T_{\mathrm{s} \rightarrow \mathrm{m}}$ did not change on the number of oxyethylene unit, while the $T_{\mathrm{m} \rightarrow \mathrm{i}}$ decreased with increasing oxyethylene unit, as shown in Figure 2. However, the mesophase became obscure when the length of oxyethylene unit was beyond six. ${ }^{6}$ It was concluded that the flexible chain length had much more influ-

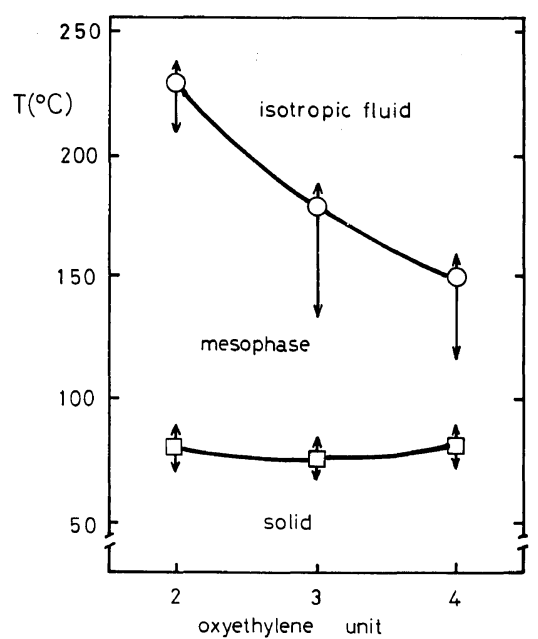

Figure 2. The dependence of the length of oxyethylene unit on the transition temperature: $(\square)$, transition temperature on melting; ( $\bigcirc$ ), transition temperature on formation of the isotropic fluid. The arrows in the figure show the temperature range in which the transition temperature varies with molecular weight.

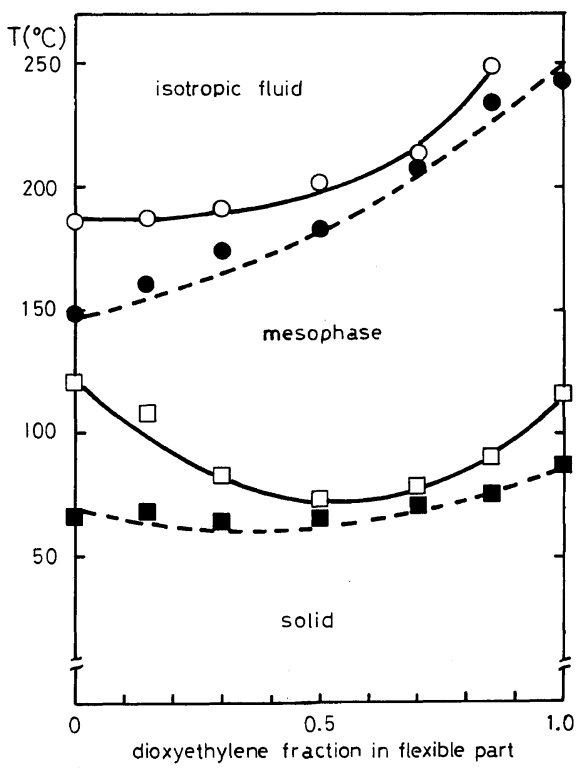

Figure 3. The transition temperature of CoPOEAOB2-4 as a function of dioxyethylene composition: ( $\square$ ), $T_{\mathrm{s} \rightarrow \mathrm{m}}$ on heating; ( $\left.\bigcirc\right), T_{\mathrm{m} \rightarrow \mathrm{i}}$ on heating ;(O), $T_{\mathrm{i} \rightarrow \mathrm{m}}$ on cooling; ( $\mathbf{\square}), T_{\mathrm{m} \rightarrow \mathrm{s}}$ on cooling.

enced on the transition temperature than the molecular weight has. 


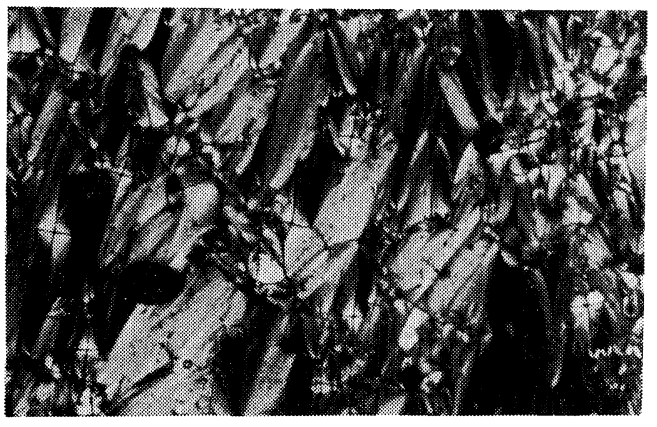

Figure 4. The polarizing microphotograph of POEAOB-4 at $130.5^{\circ} \mathrm{C}$ on cooling. Crossed polarizers, $\times 150$.

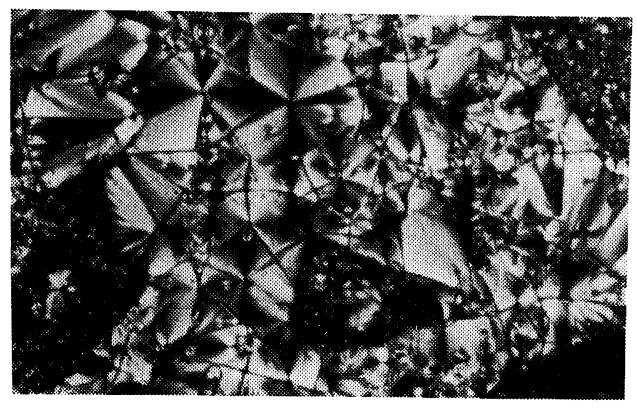

Figure 5. The polarizing microphotograph of CoPOEAOB-2-4-70 at $180.5^{\circ} \mathrm{C}$ on cooling. Crossed polarizers, $\times 150$.

Figure 3 shows the transition temperature of CoPOEAOB-2-4 as a function of di(oxyethylene) composition. The $T_{\mathrm{m} \rightarrow \mathrm{i}}$ increased with increasing di(oxyethylene) component in the copolyester, that is, the decrease in chain flexibility tended to increase the $T_{\mathrm{m} \rightarrow \mathrm{i}}$. The transition temperature revealed a minimum in the composition of a 50:50 molar ratio for di(oxyethylene) and tetra(oxyethylene) in the case of CoPOEAOB-2-4. The copolyester showed an enatiotropic mesophase for the all copolymer compositions.

The thermal stability, that is, the mesomorphic temperature range, increased also with increasing composition of di(oxyethylene) component in the copolyester. On the other hand, the mixture of homopolyesters, POEAOB-2 and POEAOB-4, in the various composition did not show a clear liquid crystalline texture, and also no significant decrease

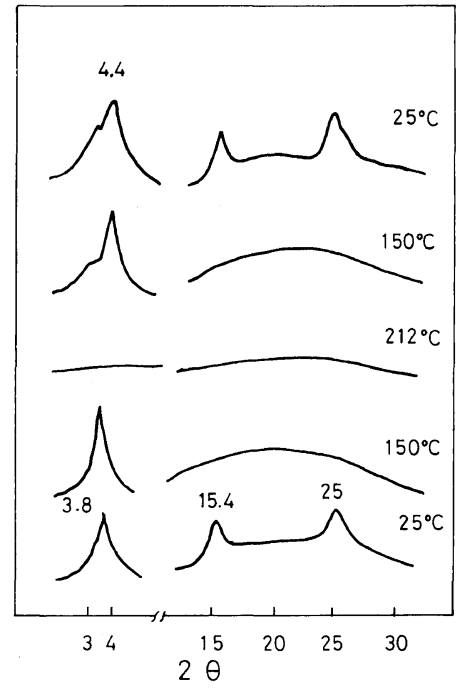

Figure 6. X-ray diffraction diagrams of the CoPOEAOB-2-4-15 at various temperatures. From bottom to uperward, the measuring temperature was risen from 25 to $212^{\circ} \mathrm{C}$ and lowered again to $25^{\circ} \mathrm{C}$.

in the $T_{\mathrm{s} \rightarrow \mathrm{m}}$ was recognized.

The optical texture of both homopolyester and copolyester were a fan-type texture which was characteristic of low molecular weight smectic liquid crystalline compounds, as shown in Figures 4 and 5.

To confirm the type of the mesomorphic phase for the copolyester, the intensity change of the X-ray diffraction peaks of CoPOEAOB2-4-15 was observed at various temperatures. The result shows in Figure 6. At $25^{\circ} \mathrm{C}$, one peak was observed at the small angle scattering region (SAXS) and two peaks were observed in the wide angle scattering region (WAXS). In the mesomorphic temperature range at $150^{\circ} \mathrm{C}$, two peaks in the WAXS disappeared and became an amorphous halo, while the sharp peak in the SAXS which corresponded to a smectic ordering remains unaltered. At the isotropic fluid state, no diffraction peaks were detected. On cooling from the isotropic fluid, two peaks appeared again in the SAXS in the mesomorphic temperature range, and the smectic orders were recovered. 
Liquid Crystalline Azoxydibenzoate Copolyesters

Table I. X-Ray measurements of CoPOEAOB-2-4-15

\begin{tabular}{cll}
\hline Temp. $/{ }^{\circ} \mathrm{C}$ & \multicolumn{2}{c}{ State $\Theta$} \\
25 & Solid $3.8(23.2)$ & $15.4(5.75) 25.0(3.56)$ \\
150 & Mesophase $3.4(26.8)$ & \\
212 & Isotropic & \\
150 & Mesophase $3.4(26.8) 4.4(20.7)$ & $15.0(5.90) 25.0(3.56)$ \\
30 & Solid $3.4(26.8) 4.4(20.7)$ & \\
\hline
\end{tabular}

a The length of periodicity $(\AA)$ is designated in parentheses.

Table II. X-Ray measurements of CoPOEAOB-2-4-70

\begin{tabular}{|c|c|c|c|}
\hline Temp. $/{ }^{\circ} \mathrm{C}$ & State & & \\
\hline 25 & Solid & $4.1(21.5)$ & $4.7(18.8) 15.3(5.79) 25.3(3.52)$ \\
\hline 150 & Mesophase & & $4.7(18.8)$ \\
\hline 232 & Isotropic & & \\
\hline 150 & Mesophase & & $4.7(18.8)$ \\
\hline
\end{tabular}

a The length of periodicity ( $\AA$ ) is designated in parentheses.

After cooling to the mesomorphic state from the isotropic fluid, two peaks appeared in the SAXS. The small anlge scatterings would have been arisen from the smectic periodicity. ${ }^{9}$ The two types of smectic arrangement would be expected. In order to obtain a more information about these structures, it was necessary to study on oriented sample.

The results of X-ray measurements for CoPOEAOB-2-4- $x$ are summarized in Tables I and II. The peaks observed at $2 \Theta=3.4^{\circ}$ for CoPOEAOB-2-4-15 and that of $4.7^{\circ}$ for CoPOEAOB-2-4-70 in the mesomorphic states correlated to the periodicities of 26.8 and $18.8 \AA$, respectively. The calculated periodicity was $19.6 \AA$ for POEAOB-2, $27.4 \AA$ for POEAOB-4, 26.2 $\AA$ for CoPOEAOB-2-4-15 and $21.9 \AA$ for CoPOEAOB-2-4-70. In the copolyester containing a small amounts of di(oxyethylene) unit $(x=15 \%)$, the periodicity estimated from the experimental data well correlated to the calculated length of the repeating unit in the extended conformation. The peak position in the SAXS shifted to lower angles, which corresponded to the longer periodicity, with increasing fraction of tetra-

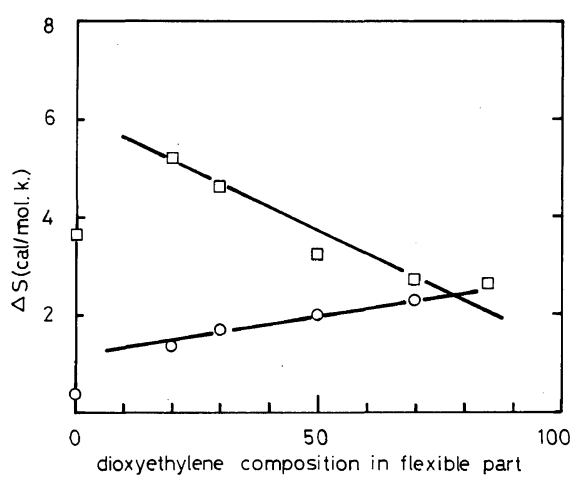

Figure 7. The transition entropy of CoPOEAOB-2-4 as a function of dioxyethylene composition: $(\square)$, entropy change from the solid to the mesophase; $(O)$, entropy change from the mesophase to the isotropic fluid.

\section{(oxyethylene).}

In the WAXS, the peak positions did not change with the fraction of di(oxyethylene). This suggested that the spacing between the two neighboring rigid molecules was nearly equal even if the flexible length in the copolyester was different.

To make sure the mesomorphic ordering of these copolyesters, we also obtained the en- 
tropy change by DSC measurements. The entropy change from the solid to the mesophase decreased with increasing content of di(oxyethylene) unit, while the entropy change from the mesophase to the isotropic fluid increased with increasing di(oxyethylene) unit, as shown in Figure 7. The change in the transition entropy from the mesophase to the isotropic fluid was ranging from 1.3 to 2.3 cal $\mathrm{mol}^{-1} \mathrm{~K}^{-1}$. In the case of conventional low molecular weight liquid crystalline materials, the entropy change from the smectic to the isotropic fluid was much larger than that from the nematic to the isotropic fluid. ${ }^{10}$ Though it was inadequate to apply the entropy change in the low molecular weight liquid crystalline materials to the liquid crystalline polymers, the order of the entropy change for CoPOEAOB-2-4 was nearly equal to the low molecular weight liquid crystalline entropy change from the smectic to the isotropic fluid. CoPOEAOB-2-4 would be ordered in a smectic arrangement, and it was suggested that the copolymer rich in the di(oxyethylene) fraction had more higher order according to the increase in entropy change.

As a parallel aggregation of the copolymer chain consisting of a constant length of the rigid rod and the different lengths of flexible chain might take place, we first expected that the orientation of the copolyesters might exhibit a nematic arrangement. However, the experimental findings by the small angle X-ray measurements, the polarized microscopic observations and the DSC measurements supported the smectic arrangement in the mesophase of CoPOEAOB-2-4.

As a smectic structure, we would like to propose a folded model in which the rigid azoxy dibenzoate rod tends to order in a parallel fashion to form lamellae, and the flexible chain forms a loose loop.
The observed smectic periodicity of $26.8 \AA$ for the copolyester having a longer flexible chain was larger than that of $18.8 \AA$ for the copolyester rich in the shorter flexible chain. In the case of the smectic ordering of the extended parallel arrangement in the copolyester, it was difficult to pack the flexible chain spacially among the mesogenic layer being arranged in a side by side fashion. The longer flexible part could not settle in the same position as the shorter flexible part. The copolyester would take a folded structure based on the above observations.

Acknowledgement. This work is supported by a grant from the Ministry of Education, Science and Culture, Japan, under Grants 455360 addressed to K.I.

\section{REFERENCES}

1. J. R. Schaefgen, T. I. Bair, J. W. Ballou, S. L. Kwolek, P. W. Morgan, M. Pavar, and J. Zimmerman, "Ultra High Modulus Polymers," A. Cifferi and I. W. Ward, Ed., Appl. Sci., London, 1979, p 203.

2. J. I. Jin, S. Antoun, C. Ober, and R. W. Lenz, Br. Polym. J., 12, 132 (1980).

3. H. Finkelmann, H. Ringsdorf, and J. H. Wendorff, Makromol. Chem., 179, 273 (1978).

4. H. Finkelmann and G. Rehage, Makromol. Chem. Rapid. Commun., 1, 31 (1980).

5. K. Iimura, N. Koide, R. Ohta, and M. Takeda, Makromol. Chem., 182, 2563 (1981).

6. Y. Kanai, N. Koide, and K. Iimura, Rep. Prog. Polym. Phys. Jpn., 26, 249 (1983).

7. H. Iida and K. Konishi, Yukikagobutsu Gohseiho, 8, 29 (1956).

8. A. Blumstein, S. Vilasagar, S. Ponrathnum, S. B. Clough, and R. B. Blumstein, J. Polym. Sci., Polym. Chem. Ed., 20, 877 (1982).

9. J. H. Wendorff, H. Finkelmann, and H. Ringsdorf, "Mesomorphic Order in Polymers and Polymerization in Liquid Crystalline Media," A. Blumstein, Ed., American Chemical Society, 1978, p 12.

10. H. Arnold, Z. Phys. Chem., 226, 146 (1964). 